\title{
Una visión holística de la reducción del impacto ambiental en edificios del área del Mediterráneo
}

\author{
A holistic view of the reduced environmental impact \\ of buildings in Mediterranean Area
}

L. Sabaté ${ }^{(*)}$, C. Peters ${ }^{(*)}$

RESUMEN

LIMA, Low Impact Mediterranean Architecture, es una iniciativa que pretende demostrar la viabilidad tecnológica y económica de reducir drásticamente el impacto ambiental de las edificaciones en países de climas cálidos, aplicable tanto a obra nueva como a rehabilitación, mejorando al mismo tiempo su confort y habitabilidad. La visión holística del impacto ambiental aquí presentada incluye no solo el consumo de energía en la fase de uso del edificio, sino también la energía incorporada en los materiales de construcción en todo el ciclo de vida del edificio así como el ciclo del agua y aspectos de salud y confort. La metodología de verificación se basa en la definición y análisis de un edificio colectivo representativo y la construcción de un prototipo, parte ficticia del edificio total, para su análisis y monitorización, tanto en condiciones estandarizadas, como reales de uso.

$113-118$

Palabras clave: materiales de construcción de bajo impacto - energia incorporada - energia en fase de uso - monitorización de prototipo.
SUMMARY

LIMA, Low Impact Mediterranean Architecture, is an initiative that aims to demonstrate the technical feasibility and economic viability of drastically reducing the environmental impact of buildings in countries with warm climates. The initiative is applicable to both new construction and torefurbishment and leads to improvements in comfort and indoor quality. The holistic vision of the environmental impact presented here contemplates notonly the energy consumed in use but also the energy and water used in the complete life cycle of the building, including the energy embodied in the materials, as well as aspects relating to health and comfort. The verification methodology is based on the definition and the analysis of atypical, reference, block of flats, the actual construction of a prototype representative of a substitution of one flat within this reference block, and the monitoring and analysis of this prototype in standard conditions and also when actually occupied.

Key words: low impact building materials - embodied energy - energy in use - building prototype monitoring. 


\section{INTRODUCCIÓN}

Existe un amplio consenso en el mundo científico sobre la contribución de las actividades humanas al cambio climático. Los cuatro informes presentados por el Panel Intergubernamental sobre Cambio Climático (IPCC) (1), en los últimos veinte años, permiten contrastar los efectos antrópicos sobre el calentamiento global del planeta, y los riesgos que se derivan si no se modifican las condiciones del actual modelo de desarrollo. Una de las conclusiones más claras es la necesidad de reducir las emisiones de $\mathrm{CO}_{2}$ y otros gases de efecto invernadero (GEI) englobados bajo el índice $\mathrm{CO}_{2 e q}{ }^{1}$ a niveles anteriores a la época industrial. Stern, N. (2007) ha planteado la necesidad de que las medidas para reducir las emisiones de $\mathrm{CO}_{2 \text { eq }}$ se adopten e implementen en la presente década (2) con el objetivo de evitar una crisis económica y social sin precedentes.

La mejora de la eficiencia en el sector de la edificación resulta clave para la reducción global de emisiones de $\mathrm{CO}_{2 \text { eq }}$. En estos momentos se lo considera responsable del $40 \%$ del consumo final de energía de la UE, con una tendencia a incrementarse en los próximos años (3). Del mismo modo este sector necesita incorporar estrategias de mitigación, que permitan mantener la habitabilidad de los edificios una vez se produzcan los efectos del cambio climático, especialmente el incremento generalizado de las temperaturas,con episodios de olas de calor, y la reducción de los recursos hídricos.

En los últimos años en el ámbito de la UE se ha desarrollado un esfuerzo encaminado a la reducción del impacto ambiental de los edificios, que se ha traducido en la reducción del consumo energético de las nuevas edificaciones, y en menor medida en la rehabilitación energética. Pero en cualquier caso esta reducción se ha limitado a los consumos durante la vida útil de los edificios sin referirse a la energía necesaria para su construcción y mantenimiento, la denominada energía incorporada.

A modo de ejemplo el Plan Nacional de Asignaciones de Derechos de Emisión 20082012 del Gobierno español, atribuye a los sectores domestico y terciario el $20 \%$ y el $8 \%$ respectivamente, de los consumos finales de energía, que representan algo más del 30\% de emisiones. Las emisiones debidas a la fabricación de los materiales como el acero, el cemento, la cerámica o el vidrio, disponen de asignaciones específicas en el mismo documento, de modo que no quedan reflejadas en el sector de la edificación.
El esfuerzo de reducción de consumos ha estado liderado por los países más desarrollados, situados básicamente en el hemisferio norte, y especialmente por los países del norte y centro de Europa, y sólo en fechas más recientes por los EEUU. Esta circunstancia explicaría la razón por la que las tecnologías de mejora de la eficiencia de las edificaciones se han centrado en la reducción de consumos debidos a la calefacción y la producción de agua caliente sanitaria (ACS), en la mejora de los sistemas de iluminación y en la producción de calor y electricidad a través de fuentes renovables, y no han abordado con la misma intensidad el desarrollo de las tecnologías adecuadas para los países templados y cálidos, en los cuales a los problemas anteriormente citados, se superponen problemas específicos como el consumo debido a la refrigeración estival o la reducción del consumo de agua para hacer frente a la reducción del régimen hídrico.

En el caso de la región mediterránea, el IPCC (4) prevé una disminución de las precipitaciones que puede alcanzar el $20 \%$ para finales del siglo, con un notable incremento de los periodos de calor extremo.

Por otra parte cabe señalar que para lograr una reducción neta de emisiones de $\mathrm{CO}_{2 \mathrm{eq}}$ no basta con la mejora de las nuevas edificaciones, sino que los esfuerzos más importantes deberán de aplicarse en la rehabilitación energética de los edificios existentes. La Decisión 406/2009/CE del Parlamento y Consejo Europeo fija la intensidad de reducción de emisiones de $\mathrm{CO}_{2 \text { eq }}$ para el año 2020 respecto a 2005, para cada país, siendo para España del $10 \%$. Para alcanzar este objetivo deberá de incrementarse la tasa de rehabilitación anual hasta el 2,5\%, (más de 300.000 viviendas anuales) con una reducción media del consumo de energía consumida debida al uso (no sólo de calefacción) posterior a la rehabilitación superior al 45\%.

En los próximos años será necesario desarrollar una estrategia viable de reducción del impacto de las edificaciones que incluya el ciclo de vida de los edificios, considere la situación específica de los países cálidos, e incluya como prioridad la rehabilitación energética y ambiental.

\section{LIMA, LOW IMPACT MEDITERRANEAN ARCHITECTURE-OBJETIVOS}

El proyecto LIMA se presentó en la feria de Contrumat 2009, en Barcelona, bajo el epígrafe de "Esto no es una casa... es una herramienta para transformar el futuro". Se trata de una iniciativa que pretende mostrar la viabilidad tecnológica y económica de reducir drásticamente el impacto de las edificaciones residenciales del área del Mediterráneo, y por

\footnotetext{
de otros gases (metano, oxid del $\mathrm{CO}$

Para poder utilizar un solo denominador para medir el conjunto de los GEI, se utiliza el termino $\mathrm{CO}_{2 \mathrm{ec}}$ que adapta el potencial de efecto

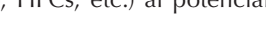


extensión de los países de climas templados similares, y verificar mediante ensayos empíricos su funcionamiento.

La iniciativa ha sido posible gracias a la participación de casi cuarenta empresas del sector de la construcción, pertenecientes a diversos países de la Unión Europea, que han apostado por un trabajo de investigación e innovación en los métodos de construcción. Este proyecto ha contado también con el soporte del Departament de Territori i Sostenibilidad de la Generalitat de Catalunya, y el apoyo financiero de la Obra Social de la Caixa de Pensions de Barcelona. Así mismo ha obtenido el premio de Medioambiente de la Generalitat de Catalunya 2009, y el Premio Agenda 21, 2010, del Ayuntamiento de Barcelona.

El proyecto plantea el desarrollo de un estándar de edificación, que tienda al cierre de los ciclos naturales de la materia, el agua y la energía, y que incorpore medidas para incrementar la salud y el confort de sus usuarios, estos dos conceptos agrupados bajo el termino de biohabitabilidad. Este sistema debería de ser adecuado para la construcción y rehabilitación de viviendas, tanto privadas como en régimen de protección pública, y por extensión de equipamientos como escuelas y otros de características similares.

Los cuatro ámbitos de actuación son:

1. Materia. Análisis del Ciclo de Vida completo del edificio (con evaluación de indicadores de energia incorporada [PEI], emisiones de $\mathrm{CO}_{2 \text { eq }}$ [GWP] y lluvia ácida [AP]) y uso de materiales renovables (biosfera), reciclados i reciclables.

2. Energia. Análisis de los consumos de energia en fase de uso (calefacción, refrigeración, ACS, iluminación, electrodomésticos y equipos) y producción a partir de fuentes renovables. Emisiones totales de $\mathrm{CO}_{2 \text { eq }}$ debidas al uso y la construcción.

3. Agua. Análisis del ciclo del agua: reducción de consumo, captación de aguas de Iluvia, reciclaje de aguas grises y negras.

4. Biohabitabilidad: Confort interior, campos eléctricos y electromagnéticos, calidad del aire, compuestos orgánicos volátiles [COV] y radioactividad natural.

En cada uno de estos campos los objetivos se centran en la definición de indicadores y estándares alcanzables, así como en la obtención de ratios fiables de coste-beneficio de cada acción de mejora mediante una visión holística del ciclo de vida del edificio.

\section{METODOLOGÍA DEL ESTUDIO}

Para lograr estos objetivos LIMA ha definido un modelo teórico, que ha sido analizado con detalle, para posteriormente construir un prototipo que esta siendo sometido a un exhaustivo ensayo. El estudio se desarrolla en las siguientes fases:

1. Definición de un modelo de edificación de carácter residencial [que denominamos LIMA 12] que pueda considerarse significativo de las edificaciones residenciales en el área de estudio, y establecimiento una línea de base correspondiente al mismo edificio realizado con sistemas constructivos, de instalaciones convencionales.

2. Evaluación de los impactos durante todo el ciclo de vida de los cuatro ámbitos enunciados: materia, energía, agua y aspectos asociados a la biohabitabilidad.

3. Definición y construcción de un prototipo experimental para comprobar de modo empírico los resultados analíticos de la fase anterior.

4. Preparación del proceso de monitorización y toma de datos.

5. Comprobación experimental de los resultados obtenidos en la fase de análisis a través de la monitorización de los indicadores de energía y agua, en dos situaciones:

a. monitorización del prototipo en condiciones estándar, obtenidas mediante la incorporación de cargas reales equivalentes a las del uso del edificio y monitorizadas en situaciones de verano e invierno, en un emplazamiento provisional en la Escuela de Arquitectura e Ingeniería de La Salle, Universidad Ramon Llull, Barcelona

b. monitorización del prototipo en condiciones reales de uso, en su emplazamiento definitivo, en un lugar cercano a Barcelona

Las fases 1, 2 y 3 se completaron durante 2008 y 2009, y la fase 4 en 2010. La fase 5-a que incluye la recogida y el análisis de datos de la experimentación relativos al ciclo de energía y agua se desarrollará durante 2011 y 2012. La fase 5-b, con mediciones experimentales referentes a biohabitabilidad y la aplicación de distintas tecnologías de inercia térmica estacional, ciclo de agua y cubiertas vegetales en base a un uso real de la vivienda está prevista que se inicie a finales de 2012 y finalice en 2014.

Para definir el modelo de análisis se tomó como referente una promoción de 60 viviendas, en régimen de protección oficial (VPO) en Tossa de Mar, proyectadas por el estudio de arquitectura Sabaté associats (SaAS) para el Instituto Catalán del Suelo (INCASOL). Se trata de un proyecto de viviendas de bajo impacto, en el que se logra una reducción de emisiones de $\mathrm{CO}_{2 \text { eq }}$ superiores al $45 \%$, y que 
había servido anteriormente como referencia para una investigación sobre las emisiones de $\mathrm{CO}_{2 \text { eq }}$ producidas a lo largo de un ciclo de vida de 50 años (5). Por estas razones se disponía de datos analizados, tanto del proyecto realizado como de la línea base, definida por un edificio de referencia que cumpliera estrictamente CTE.

A partir de este proyecto se creó un edificio teórico, con una geometría más simple, y se mejoraron sus características constructivas para alcanzar los objetivos de reducción de
El prototipo reproduce una parte (ficticia) del edificio total: la zona de día de una vivienda situada en el extremo del bloque, que dispone al mismo tiempo de cubierta y plano de contacto con el terreno. Este edificio prototipo se simuló en las mismas condiciones y con idéntico programa, y una vez obtenidos los resultados estos se compararon con los del edificio LIMA 12. La relación entre los dos datos permitió establecer una correspondencia escalar que permitirá traspasar datos y resultados de uno a otro modelo. El esquema de trabajo sigue el siguiente gráfico:

\section{LIMA - Low Impact Mediterranean Architecture}

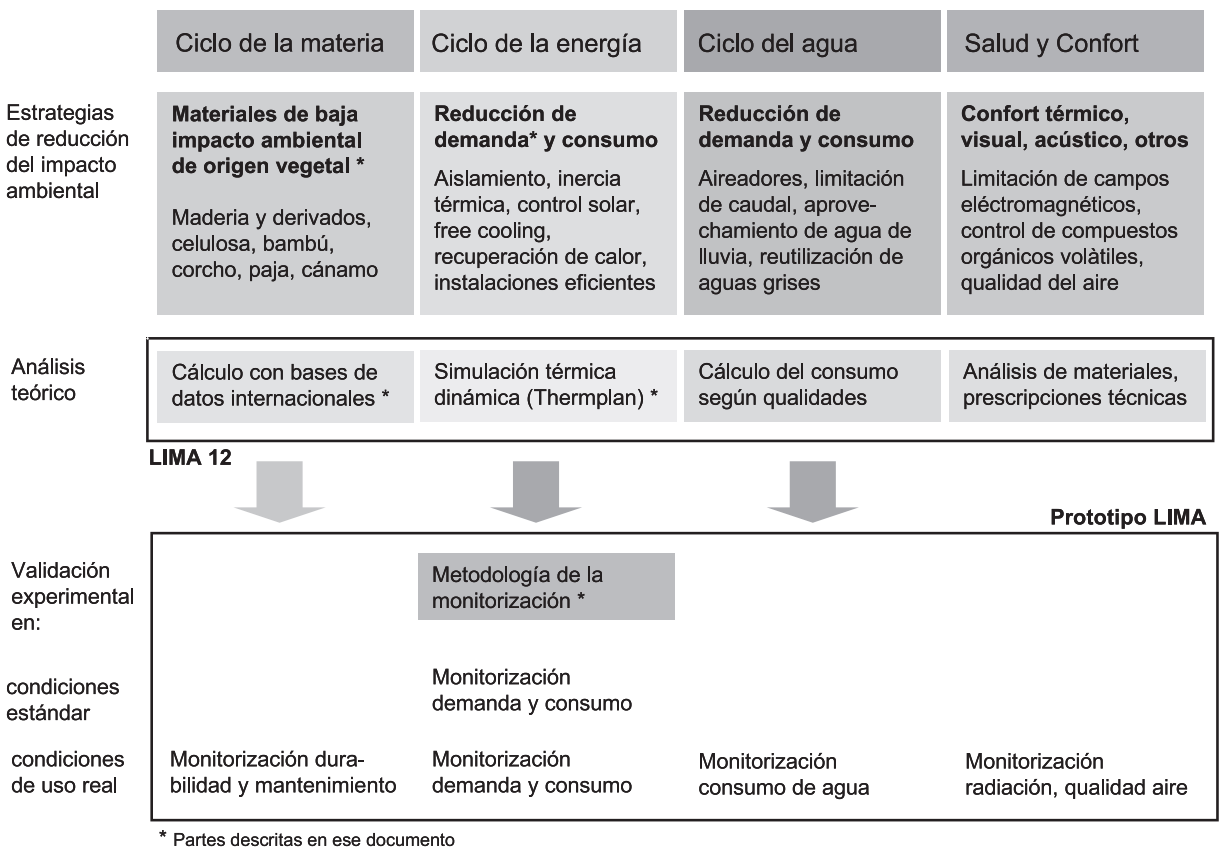

impacto. Este nuevo edificio, denominado LIMA 12, fue el que se analizó a lo largo de todo el proceso de elaboración del estudio y cuyos datos finales se presentan en este artículo.

Una vez definido el proyecto del modelo LIMA 12 y obtenidos los datos teóricos, se diseño y construyó un prototipo para verificar en condiciones reales el cumplimiento de algunos de los datos obtenidos mediante simulación.

En la primera fase (5-a) se comprobará la demanda y consumo energético en fase de uso, el funcionamiento de la envolvente térmica y de los sistemas técnicos de climatización. En la segunda (5-b) se analizaran, además, el ciclo completo del agua, los sistemas de inercia estacional y algunos datos referentes a la biohabitabilidad, como campos eléctricos y electromagnéticos, y COV.

\section{DEFINICIÓN DE LAS CARACTERÍSTICAS DEL EDIFICIO LIMA}

\subsection{Tipologia edificatoria}

El edificio estudiado es un pequeño bloque de 12 viviendas desarrolladas en cuatro plantas, con una superficie útil $\left(\mathrm{S}_{\mathrm{u}}\right)$ de $75,41 \mathrm{~m}^{2}$ por vivienda. Para la orientación se tomó la misma que en el proyecto de Tossa de Mar, con fachadas principales a SE i NO. El porcentaje de aperturas respecto fachada opaca es de $18,65 \%$, que se considera una fracción razonable para garantizar el confort y fomentar el ahorro energético. El edificio cumple tanto el CTE, como la normativa de habitabilidad de Cataluña, más exigente en lo que se refiere a dimensiones mínimas y accesibilidad que los estándares medios.

Desde el punto de vista del programa y superfície, LIMA 12 representa una tipologia 
media, ya que en Cataluña de los 2,3 millones de viviendas existentes (2001) un 45\% se situaba en el rango de superficies de 60 y 89 $\mathrm{m}^{2}$ superficie útil. Tipológicamente presenta algunas singularidades con respecto de las edificaciones habituales como son la eliminación de la planta sótano y el acceso por pasarela exterior.

La eliminación de la planta sótano dedicada a aparcamiento, presente en el proyecto original de Tossa de Mar, es una de las recomendaciones del estudio citado sobre reducción de emisiones de $\mathrm{CO}_{2 \text { eq }}$ en vivienda pública, debido al importante impacto ambiental que implica la utilización intensiva de hormigón armado. Se propone sustituir en las zonas de nueva construcción, el tradicional aparcamiento bajo las viviendas por edificios específicos en altura, que incluyan además otros equipamientos de escala de barrio. Esta propuesta se basa en experiencias similares desarrolladas en países del centro y norte de Europa.

El acceso a través de pasarelas exteriores, si bien es poco habitual en nuestro país, constituye una buena solución en tanto que limita el número de núcleos verticales de acceso y la superficie construida, y es la utilizada en el proyecto de Tossa de Mar.

La definición de los materiales y sistemas constructivos, así como las estrategias respecto de la energía, con sistemas pasivos o activos, se desarrollaron de modo interactivo con los resultados de los análisis. Se definieron los sistemas constructivos atendiendo tanto a los impactos debidos a la fabricación a lo largo de su ciclo de vida, como de la eficiencia energética durante la fase de uso, y las condiciones respecto de la salud y confort de los usuarios. Las instalaciones se definieron también en base a factores de eficiencia (COP), salud y confort.

\subsection{Sistemas constructivos}

Las fachadas están formadas por paneles de madera contralaminada de $78 \mathrm{~mm}$ de abeto (con $0,4 \mathrm{Kg} / \mathrm{m}^{2}$ de adhesivo de poliuretano [PUR]), revestimiento interior de placas de celulosa-yeso de 12,7 mm sobre rastreles de madera y acabado con pintura mineral de sol-silicato. El aislamiento se situa en el exterior y está formado por paneles de $120 \mathrm{~mm}$ de fibras de madera (4\% de PUR), lámina semipermeable de polipropileno (PP), cámara de aire de $40 \mathrm{~mm}$, y acabado exterior de lamas de alerce de $19 \mathrm{~mm}$, con tratamiento de aceites naturales. $\left(\mathrm{U}=0,26 \mathrm{~W} / \mathrm{m}^{2} \cdot \mathrm{K}\right)$.

Las carpinterías disponen de marcos de madera laminada de alerce y acristalamien-
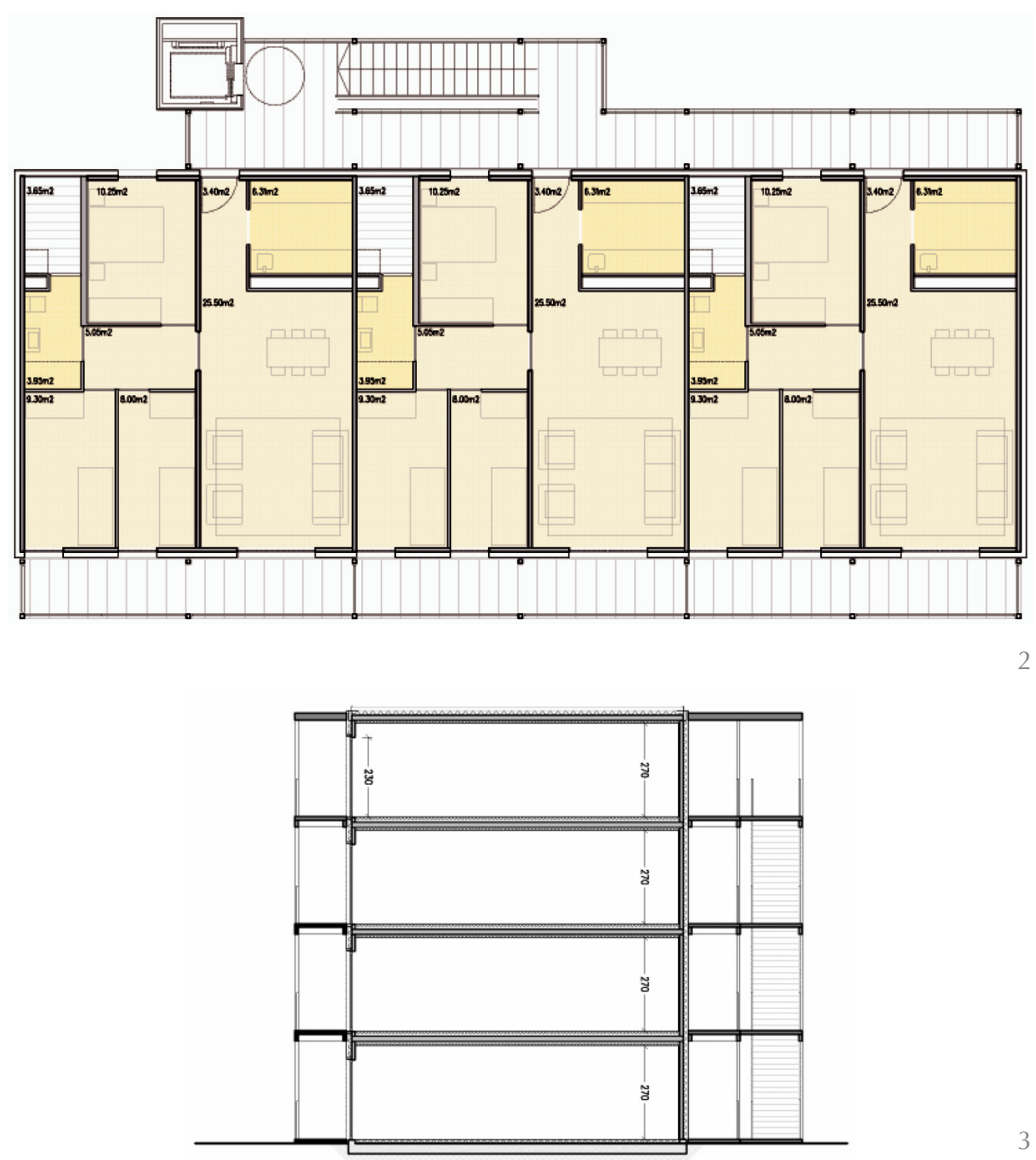

to $6 / 15 / 6$, con capa bajo emisiva en la cara 2 y argón $90 \%$ en la cámara $\left(U=1,1 \mathrm{~W} / \mathrm{m}^{2} \cdot \mathrm{K}\right.$; factor solar $\mathrm{g}=0,48)$. (U global =1,4 W/m² $\cdot \mathrm{K})$.

2. Planta edificio LIMA 12.

3. Sección edificio LIMA 12.

Las protecciones solares son de lamas exteriores de aluminio apilables y orientables, con doble obertura en la parte superior para transportar la luz solar al interior, accionadas automáticamente según posición solar y luminosidad.

Las cubiertas vegetales extensivas, tienen una plantación de Sedum, sobre $80 \mathrm{~mm}$ de compost, placa de drenaje y retención de agua $\left(24 \mathrm{lts} / \mathrm{m}^{2}\right)$ y láminas filtrantes de PP-PE, e impermeabilización de EPDM. La estructura es de paneles de madera contralaminada de abeto, de $117 \mathrm{~mm}\left(0,8 \mathrm{Kg} / \mathrm{m}^{2}\right.$ de PUR), y dispone de un aislamiento superior de paneles de $120 \mathrm{~mm}$ de fibra de madera (4\% de PUR) y barrera de vapor de difusión variable de polipropileno (PP). El acabado interior se realiza con placas de celulosayeso de 12,7 mm sobre rastreles de madera y acabado con pintura mineral de sol-silicato. $\left(\mathrm{U}=0,25 \mathrm{~W} / \mathrm{m}^{2} \cdot \mathrm{K}\right)$

El forjado inferior es de paneles de madera contralaminada de abeto, de $117 \mathrm{~mm}$ $\left(0,8 \mathrm{Kg} / \mathrm{m}^{2}\right.$ de PUR), con aislamiento de 100 $\mathrm{mm}$ de fibras de madera ( $4 \%$ de PUR) con parquet de tarima de bambú de $15 \mathrm{~mm}$. $\left(\mathrm{U}=0,36 \mathrm{~W} / \mathrm{m}^{2} \cdot \mathrm{K}\right)$ 
4. Sección constructiva.
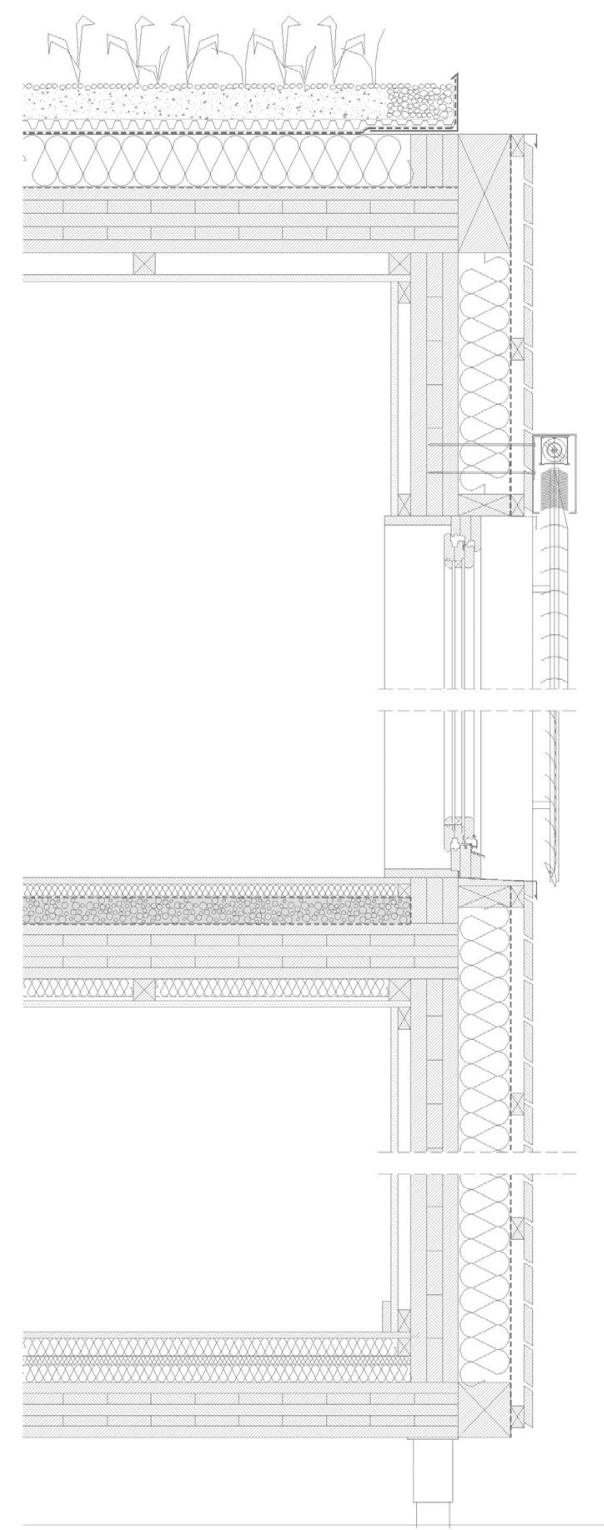

Los forjados intermedios son de paneles de madera contralaminada de abeto, de $125 \mathrm{~mm},\left(0,9 \mathrm{Kg} / \mathrm{m}^{2}\right.$ de PUR), aislamiento superior de $30 \mathrm{~mm}$ de fibras de madera (4\% de PUR) y $60 \mathrm{~mm}$ de balasto (para incrementar inercia térmica y mejorar aislamiento acústico) y fieltro (PP) inferior y superior. El pavimento es de tarima de bambú de $15 \mathrm{~mm}$. En la cara inferior se dispone un aislamiento de celulosa de $40 \mathrm{~mm}$, con revestimiento de placas de celulosa-yeso de 12,7 mm, sobre rastreles de madera, y acabado con pintura mineral de sol-silicato.

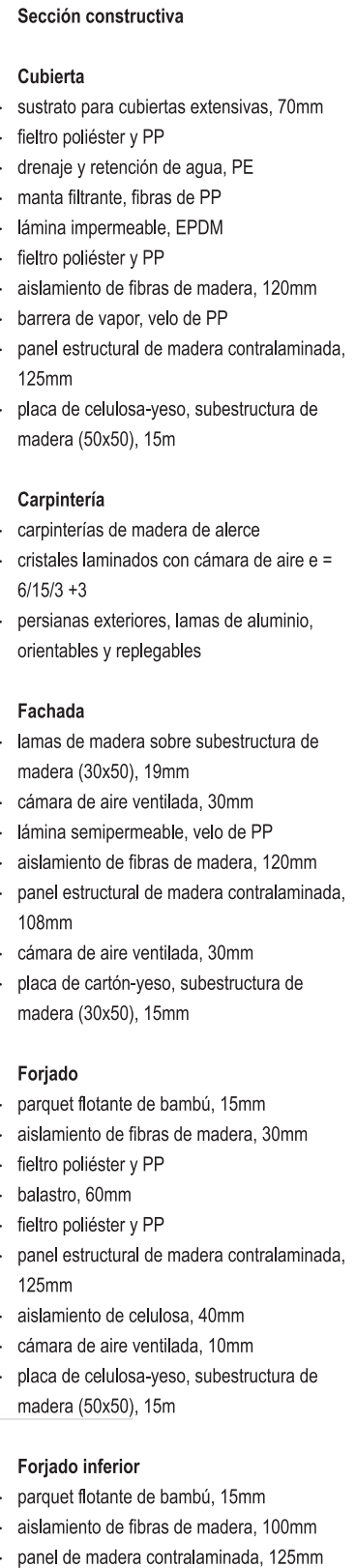

\section{ANÁLISIS DE LOS IMPACTOS - MATERIA}

\subsection{Descripción de las estrategias de reducción de impacto ambiental}

Para evaluar el impacto de los materiales y procesos constructivos del edificio se realizó un análisis de ciclo de vida (ACV) del mismo, que incluía la construcción (extracción, manufactura de los materiales, transporte y puesta en obra), las reposiciones durante la vida útil, y los procesos de reciclaje y valoración una vez concluida esta. Este ACV se limitó a tres indicadores: energía incorporada (PEI), emisiones de $\mathrm{CO}_{2 \text { eq }}(\mathrm{GWP})$ y lluvia ácida (AP), con especial atención a los dos primeros debido a su incidencia en el cambio climático.

La realización de ACV resulta aún poco habitual en el sector de la edificación. La reciente publicación del informe Cambio Global España 2020/50 resume en palabras claras la situación actual en este ámbito (6): "A pesar del desconocimiento que existen sobre este impacto, y de la necesidad de producir información y ponerla al alcance de los agentes del sector, se trata de una línea de trabajo donde se puede lograr grandes adelantos, justamente porque no ha sido nunca identificada como un impacto clave de la edificación".

Sabaté, J., et al. (5) muestran como las emisiones de $\mathrm{CO}_{2 \text { eq }}$ debidas a la fabricación de los materiales de construcción, pueden llegar a representar entre el 30 y el $45 \%$ de toda la energía consumida por un edificio en su ciclo de vida. Como materiales de mayor impacto ambiental se identifican los metales, el cemento y la cerámica. En el caso del hormigón armado las emisiones de $\mathrm{CO}_{2 \text { eq }}$ llegan a alcanzar cerca del $50 \%$ del total de emisiones debidas a la fabricación de materiales.

Los resultados de este estudio divergen de análisis de ciclo de vida de los edificios, como los presentados por Winther y Hestnes (7) en los que en ninguno de los edificios estudiados la energía incorporada es superior al 10\% de la energía global. En un estudio comparativo posterior de sesenta edificios, mayoritariamente residenciales y todos de países nórdicos (a excepción de seis casos de Australia y Nueva Zelanda y tres de Estados Unidos donde la climatología queda indefinida), Sartori, I. y Hestnes, A.G. (8) identifican una fracción de energía incorporada entre 2 y $38 \%$ para edificios convencionales y 9 a $46 \%$ para edificios de bajo consumo energético, más cercano a los resultados del estudio de Tossa de Mar.

Feist, W. fundador del Instituto Passivhaus, muestra una fracción de energía incorporada 


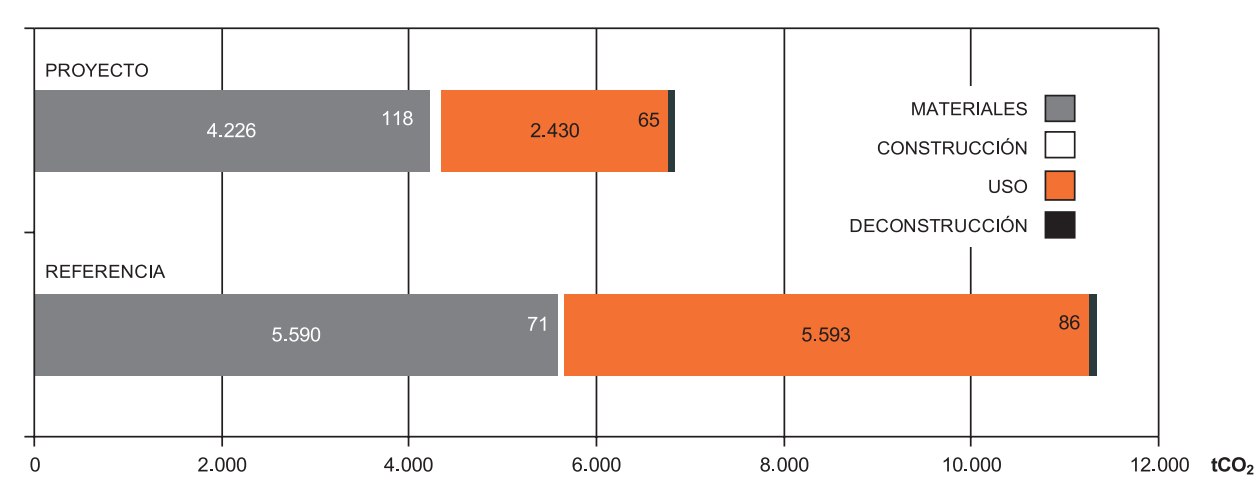

muy reducida en edificios convencionales, pero detecta un importante aumento de esta fracción hacia el consumo global de energía en edificios construidos en estándar Passivhaus, con fracciones de hasta un 32\% (9). Este aumento se debe principalmente a la radical reducción de la energía necesaria para la fase de uso del edificio (condición estándar Passivhaus: demanda de calefacción inferior a $15 \mathrm{~kW}_{\text {energia útil }} / \mathrm{m}^{2} \cdot$ año y consumo global para calefacción, refrigeración, ACS e iluminación inferior a $120 \mathrm{~kW}_{\text {energia primàriai }} / \mathrm{m}^{2} \cdot$ año).

Las diferencias en estos estudios pueden ser debidas a distintos factores. Por una parte todos los ejemplos estudiados a excepción de Tossa de Mar se encuentran en regiones frías, en las que la energía necesaria para la calefacción puede fácilmente triplicar o cuadriplicar su equivalente en las regiones mediterráneas. Por otra parte los modelos constructivos españoles, en especial tras la aprobación del CTE y la reglamentación del hormigón armado (EHE), apuestan por el uso intensivo del hormigón con importantes cuantías de cemento para garantizar la durabilidad, muy superior a las exigencias en otros países de estudio, hecho que incide en el incremento de la energía incorporada.

Para reducir el impacto debido a los materiales se analizaron diversas opciones: utilizar materiales de baja energía incorporada y bajo impacto medioambiental, utilizar materiales reciclados y/o fácilmente reciclables o utilizar materiales procedentes de la biosfera. La última opción presenta una ventaja adicional respecto a las primeras ya que además de requerir poca energía para su transformación, representa el secuestro de $\mathrm{CO}_{2 \text { eq }}$ absorbido durante el crecimiento del vegetal y que permanece fijado tanto en la madera comercializable como en sus raíces que se integran al suelo.

Existen algunas controversias sobre la consideración de la construcción en madera como sumidero de carbono. La opción que toma este trabajo es la de considerar que la utilización de la madera en la construcción, siempre que su duración supere el ciclo natural de crecimiento de la especie y la madera proceda de una explotación sostenible, constituye un claro secuestro de las emisiones de $\mathrm{CO}_{2 \text { eq }}$ realizadas por el árbol en su crecimiento, y que en el caso de no ser utilizado retornarían al ciclo natural del carbono, ya sea por la quema accidental (convertidos en $\mathrm{CO}_{2}$ ) ya por su pudrición al finalizar su vida (convertidos en metano).

En la elección del uso de materiales vegetales se tuvo en consideración las conclusiones del estudio de Sabaté, J. et al. (10) sobre las emisiones de $\mathrm{CO}_{2 \text { eq }}$ a partir de diversos sistemas constructivos y de instalaciones, desde los más convencionales a los más eficientes en el uso de los recursos. Según este estudio la construcción con materiales renovables de la biosfera permite alcanzar reducciones del $90 \%$ en las emisiones de $\mathrm{CO}_{2 \mathrm{eq}}$ debidas a la energía incorporada, que pasaban de los $55 \mathrm{Kg}$ de $\mathrm{CO}_{2 \mathrm{eq}} / \mathrm{m}^{2}{ }_{\text {su }}$. año de un edificio construido con sistemas anteriores al CTE (2005) hasta 5,5 $\mathrm{Kg}$ de $\mathrm{CO}_{2 \text { eq }}$ $/ \mathrm{m}^{2}{ }_{\mathrm{su}} \cdot$ año de una vivienda de "factor $10^{\prime \prime}$. La reducción más significativa se alcanzaba mediante la sustitución del sistema estructural de hormigón armado por una estructura de madera.

El uso de la madera como material estructural y de construcción tiene pocos precedentes en el Mediterráneo, una región en la que tradicionalmente ha imperado una construcción con materiales minerales. Históricamente la madera se reservó a las estructuras flexionadas, que no pueden resolverse con materiales pétreos, como los techos y cubiertas. La introducción del hormigón armado como material estructural, a comienzos del s. $x x$, desplazó completamente el uso de la madera en el ámbito de las estructuras. Sólo en ciertas regiones de Turquía, incluida su capital Estambul, existen edificaciones tradicionales enteramente construidas con madera, similares a las de las regiones más septentrionales.
5. Emisiones de $\mathrm{CO}_{2}$ incorporadas a los materiales y debidas a la fase de uso del edificio, ejemplo residencial para un proyecto que cumple el CTE y un proyecto mejorado. 
6. Emisiones de $\mathrm{CO}_{2 \text { eq }}$ y costos de diferentes modelos de construcción, Sabaté, J.

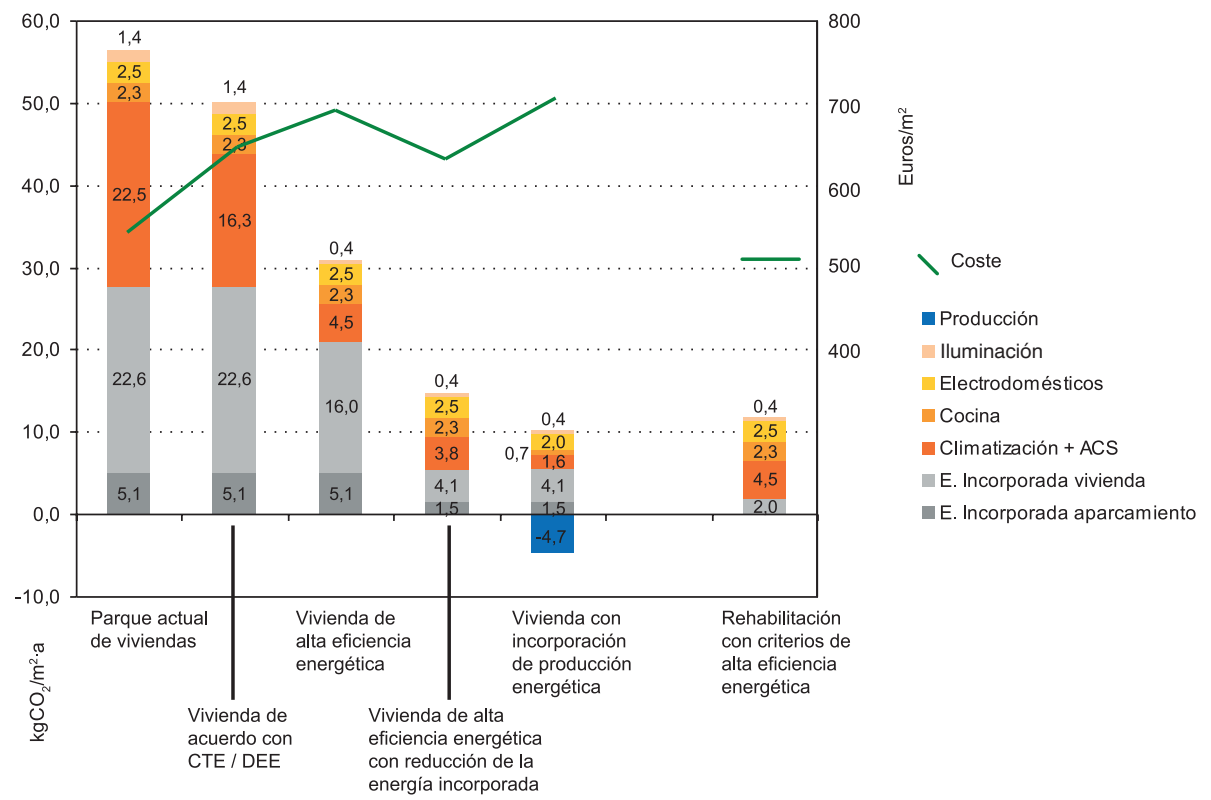

La tecnología actual de industrialización de la madera, junto con sus ventajas ambientales, pueden facilitar una nueva concepción de la madera en la construcción en climas cálidos. Los nuevos modelos de prefabricación en madera a base de grandes paneles de entramado ligero o madera contralaminada, realizados a partir de sistemas de corte numérico, permiten realizar edificios a medida al mismo coste que series completas. Las características estructurales de la madera, que resiste esfuerzos de compresión y tracción, su bajo peso y la facilidad de unión y montaje, así como los valores ambientales en cuanto a reducción de energía incorporada y emisiones de $\mathrm{CO}_{2 \text { eq }}$ le otorgan una gran competitividad en la construcción contemporánea capaz de desplazar un material como el hormigón armado, que pese a su corta historia de algo más de un siglo, se ha transformado en el estándar indiscutible de la construcción moderna, aunque como se dijo anteriormente a costa de un gran impacto ambiental.

Yates, M. et al. (11) muestran que con un adecuado diseño constructivo y estructural, las construcciones de muros y techos de madera contralaminada pueden resolver satisfactoriamente edificios de 6 a 10 pisos de altura, contra todos los perjuicios como una falta de resistencia al fuego, contra movimientos térmicos y una reducida durabilidad.

\subsection{Metodología de cálculo y procedencia de los datos}

La decisión sobre los materiales y sistemas constructivos del edificio modelo se tomó después de analizar un número considerable de opciones, en las que se analizaron los tres impactos antes mencionados: energía incorporada, emisiones de $\mathrm{CO}_{2 \text { eq }}$ y acidificación. El modelo de análisis se basó en la descomposición de sistemas completos, como fachadas o cubiertas, en las distintas capas que lo conforman, las cuales a su vez se refirieron a unidades de materiales de los cuales pudiésemos disponer de datos ambientales. Para cada material y cada capa se definieron características físicas (grueso, densidad), higrométricas (conductividad, difusividad, calor específico), durabilidad (número de renovaciones en 50 años), transporte (distancia y emisiones de $\mathrm{CO}_{2 \text { eq }}$ debidas al transporte), datos ambientales (PEI, GWP, AP), y económicas (coste unitario). Con estos datos de entrada se calculó peso del elemento completo $(\mathrm{Kg})$, transmitancia térmica $\left(\mathrm{W} / \mathrm{m}^{2} \mathrm{~K}\right), \mathrm{PEI}\left(\mathrm{MJ} / \mathrm{m}^{2}\right)$, $\operatorname{GWP}\left(\mathrm{Kg} \mathrm{CO}_{2 \mathrm{eq}} / \mathrm{m}^{2}\right)$, AP $\left(\mathrm{Kg} \mathrm{SO}_{2 \mathrm{eq}} / \mathrm{m}^{2}\right)$ y coste global (EUR/m²).

Estos datos sobre cada sistema constructivo se agregaron en función de las mediciones del proyecto, de modo similar a como se analizan los costes económicos totales.

Debido a la enorme complejidad del cálculo de ACV de los sistemas de instalaciones los datos referidos a estas partidas se han estimado de acuerdo a modelos agregados. No se han tenido en cuenta los impactos relacionados a la construcción misma, como la energia consumida por la maquinaria, aunque sí toda la relacionada con el transporte y el número de reposiciones en los 50 años de vida útil considerada.

Tal como se ha planteado, de todos los datos del ACV se analizan solamente los referidos a PEI, GWP y AP, ya que se trata de los indi- 
cadores de impacto ambiental más elevado y de uso más universal. La fuente de estos valores, referidos generalmente al peso del material $(/ \mathrm{kg})$, a la superficie $\left(/ \mathrm{m}^{2}\right)$ o al volumen $\left(\mathrm{m}^{3}\right)$ de producto, se han tomado de la base de datos del Swiss Federal Laboratories for Materials Testing and Research (EMPA). Estos datos han sido complementados con otros procedentes del Instituto de Tecnología de la Construcción de Cataluña (ITeC) y de los libros de Hegger, M. et al (12) y Waltjen, $\mathrm{T}$ (13). Los datos económicos provienen mayoritariamente de la base BEDEC del ITeC y de datos directos de mercado aportados por las empresas suministradoras e instaladoras del sector.

Si comparamos los resultados de los cálculos de emisiones de $\mathrm{CO}_{2 \text { eq }}$ correspondientes a la fabricación y puesta en obra de los materiales con los de Tossa de Mar, obtendremos:

\begin{tabular}{|l|r|r|r|}
\cline { 2 - 4 } \multicolumn{1}{c|}{} & \multicolumn{2}{c|}{ Tossa de Mar } & \multirow{2}{*}{ LIMA } \\
\cline { 2 - 4 } \multicolumn{1}{c|}{} & \multicolumn{1}{c|}{ CTE } & \multicolumn{1}{c|}{ proyecto } & \\
\hline $\mathrm{KgCO}_{2 \mathrm{eq}} / \mathrm{m}^{2}{ }_{\mathrm{su}}$ & $1.428,72$ & $1.028,42$ & $-46,05$ \\
\hline $\mathrm{KgCO}_{2 \mathrm{eq}} / \mathrm{m}^{2}{ }_{\mathrm{sc}}$ & 733,85 & 528,24 & $-38,10$ \\
\hline $\mathrm{KgCO}_{2 \mathrm{eq}} / \mathrm{m}^{2}{ }_{\text {su }}$ año & 28,57 & 20,57 & $-0,77$ \\
\hline $\mathrm{KgCO}_{2 \mathrm{eq}} / \mathrm{m}^{2}{ }_{\mathrm{sc}} \cdot$ año & 14,68 & 10,56 & $-0,63$ \\
\hline
\end{tabular}

\section{ANÁLISIS DE LOS IMPACTOS - ENERGIA}

La energía incorporada y las emisiones de $\mathrm{CO}_{2 \text { eq }}$ correspondientes se han incluido en el análisis de la MATERIA. En este apartado se trata la energía consumida durante el uso del edificio:

1. Climatización: calefacción, refrigeración y ventilación.

2. Producción de agua caliente sanitaria (ACS).

3. Alumbrado.

4. Electrodomésticos.

5. Cocción.

En un edificio de viviendas la mayor parte de la energía consumida en la fase de usos se utiliza para conseguir las condiciones de confort térmico ( $y$ en ocasiones higrotérmico) establecidas, denominadas condiciones de consigna. Para reducir este consumo es necesario actuar sobre tres aspectos, por este orden: reducir la demanda energética, mejorar el rendimiento de los equipos térmicos y garantizar los medios para un adecuado control. Una vez obtenida una reducción significativa del consumo, y para satisfacer la demanda restante de energía, daremos prioridad al uso de energías renovables (solar, eólica, biomasa, etc.) ya sea de forma individual o de forma colectiva a traves de redes de calor y frio urbanas.

\subsection{Descripción de las estrategias de reducción de demanda energética en el proyecto LIMA}

\subsubsection{Mejora del envolvente}

Algunos de los factores para obtener mejoras en el comportamiento del envolvente son: incrementar el aislamiento térmico, utilizar de manera eficiente la inercia térmica, gestionar correctamente ganancias por radiación (exposición y protección solar) y limitar las infiltraciones no deseadas.

La reducción de la transmitancia térmica $U$ $\left(\mathrm{W} / \mathrm{m}^{2} \cdot \mathrm{K}\right)$ de los distintos cerramientos y la eliminación de los puentes térmicos es la estrategia más rentable para reducir la demanda energética de un edificio. En las zonas cálidas como el Mediterráneo esta estrategia es útil tanto para invierno como para verano, aunque en este último caso debe de ser analizada conjuntamente con estrategias de ventilación e inercia térmica.

La siguiente tabla muestra los valores de transmitancia térmica adoptados en LIMA respecto de las exigencias del CTE:

Tabla 1

Transmitancia térmicas de LIMA y exigencias del CTE, por situación y altitud sobre nivel del mar $(\mathrm{m} / \mathrm{snm})$

\begin{tabular}{|lcccccc|}
\hline & & LIMA & CTE, zona B & CTE, zona C & CTE, zona D & CTE, zona E \\
\hline Barcelona & $\mathrm{m} / \mathrm{snm}$ & & - & $1-400$ & $401-800$ & $>801$ \\
\hline Girona & $\mathrm{m} / \mathrm{snm}$ & & - & - & $143-742$ & $>743$ \\
\hline Lleida & $\mathrm{m} / \mathrm{snm}$ & & - & - & $131-530$ & $>531$ \\
\hline Tarragona & $\mathrm{m} / \mathrm{snm}$ & & $1-200$ & $201-600$ & $601-1000$ & $>1000$ \\
\hline & & & & & & \\
\hline fachadas & $\mathrm{W} / \mathrm{m}^{2} \cdot \mathrm{K}$ & 0,26 & 0,82 & 0,73 & 0,66 & 0,57 \\
\hline cubierta & $\mathrm{W} / \mathrm{m}^{2} \cdot \mathrm{K}$ & 0,25 & 0,45 & 0,41 & 0,38 & 0,35 \\
\hline solera & $\mathrm{W} / \mathrm{m}^{2} \cdot \mathrm{K}$ & 0,36 & 0,52 & 0,50 & 0,49 & 0,48 \\
\hline coberturas & $\mathrm{W} / \mathrm{m}^{2} \cdot \mathrm{K}$ & 1,40 & $2,70-5,70$ & $2,20-4.40$ & $1,90-3,50$ & $1,90-3,10$ \\
\hline
\end{tabular}

Tradicionalmente en los países cálidos se ha utilizado la inercia térmica del edificio (construcciones masivas con materiales minerales) para combatir el sobrecalentamiento de verano, de gran eficàcia en zonas de climatologia continental, con grandes saltos térmicos día / noche. Por oposición la falta de masa ha sido una crítica habitual a la construcción en madera en estos mismos países.

LIMA incorpora inercia térmica en el exterior en la cubierta, la zona más expuesta al sol. La cubierta ajardinada extensiva, con $8 \mathrm{~cm}$ de compost y $24 \mathrm{lts} / \mathrm{m}^{2}$ de capacidad de retención de agua, actua como una pantalla contra el sobrecalentamiento, que se complementa con el efecto producido por la evapotranspiración de las plantas. La retención del agua 
se efectua sin contacto con el compost ni con las láminas de drenaje, reduciendo la evaporación en periodos de calor.

El control de la radiación solar directa permite evitar el sobrecalentamiento en verano, pero también garantizar la aportación solar en los periodos fríos. Para ello existen dos opciones: utilizar protecciones fijas horizontales en fachadas orientadas entre SE i SO (aprovechando la variación del ángulo solar), o instalar sistemas móviles con una adecuada regulación.

En el prototipo LIMA las oberturas disponen de protecciones solares exteriores formadas por lamas orientables y apilables de aluminio, accionadas automáticamente según el régimen de verano-invierno, la posición solar y la luminosidad exterior, que proporcionan una óptima protección en verano y permiten la captación en invierno. Un sistema de modificación del ángulo de orientación, en el tercio superior de las lamas, permite el transporte de la luz natural al interior del edificio evitando al mismo tiempo el sobrecalentamiento.

Las partes opacas de las fachadas incorporan una última hoja muy ventilada y aislante (madera de alerce) que actua como umbráculo del edificio. El flujo de aire que circula entre esta hoja y la cara exterior del aislamiento facilita la evacuación del calor producido por la radiación solar y permite mantener la temperatura de la cara exterior del aislamiento en un orden de magnitud similar a la del aire exterior en zonas no expuestas al sol.

Finalmente el cerramiento garantiza un grado elevado de estanqueidad al aire, para evitar las perdidas o ganancias por infiltraciones no controladas. Por eso el envolvente del edificio dispone de una lámina impermeable al aire y al agua, pero permeable al vapor de agua, así como un completo sistema de sellado de todas las juntas constructivas. Las carpinterías disponen de doble junta de estanqueidad de goma.

La estanqueidad se mide mediante un ensayo denominado blower-door, en el que se aplica una presión positiva o negativa de 50Pa en el interior del edificio respecto del aire exterior. La caída de esta diferencia de presión permite evaluar las infiltraciones debidas a los cerramientos. El estándar Passivhaus pide un ratio de infiltraciones ${ }^{2}$ inferior a $0,6 \mathrm{~h}^{-1}$ a esta presión. Obtener este nivel de estanqueidad implica renunciar a algunas opciones, como el disponer de ventanales correderos, que resultan menos justificadas en países cálidos en los que la relación interior-exterior es
Ford, B. et al. (14) proponen en el marco del proyecto europeo Passive-on, que busca una adaptación del estándar Passivhaus a climas europeos templados, reducir esta exigencia a ratios de $1,0 \mathrm{~h}^{-1}$ en climatologías donde la temperatura exterior de diseño sea superior a $0^{\circ} \mathrm{C}$, ya que sería suficiente para cumplir las exigencias de demandas máximas de calefacción establecidas por el estándar. Peper, S. (15) ha estudiado la importancia de la estanqueidad al aire, problemas, soluciones y su durabilidad en edificios Passivhaus en una gran cantidad de ejemplos de edificios construidos en ese estándar.

\subsubsection{Ventilación}

Todo edificio requiere de una mínima ventilación, ya sea para garantizar la calidad del aire interior, evacuar el exceso de humedad debida a actividades en cocina o baño, o diluir las emisiones de compuestos orgánicos volátiles (COV) de materiales y mobiliario. En edificios con un buen nivel de aislamiento térmico la ventilación representa la mayor fuente de pérdidas (o ganancias) de calor, y por tanto de consumo energético. Cuando esta ventilación se realiza sin un pre tratamiento del aire es también una notable fuente de disconfort.

Para garantizar la calidad del aire adecuada al uso y reducir las perdidas (o ganancias) debidas a esta renovación, LIMA incorpora una ventilación mecánica con recuperación de calor, que se acciona en función de la ocupación. El mecanismo de regulación garantiza una mínima renovación de $0,2 \mathrm{~h}^{-1}$, en situación de no ocupación, de hasta $1^{\text {h-1 }}$ en régimen de ocupación y de hasta $4^{\text {h-1 }}$ en posición de enfriamiento por free-cooling. El aire interior se extrae de los espacios más contaminados, cocina y baño, y a través de un recuperador entálpico, en el que éste cede el calor (en régimen de invierno) al aire de renovación, se expulsa al exterior. Los rangos de recuperación comprobados son notables, en régimen de invierno con temperaturas exteriores de $6^{\circ} \mathrm{C}$ e interiores de $20^{\circ} \mathrm{C}$, se logran ingresos de aire a $18^{\circ} \mathrm{C}$ sin necesidad de aportaciones adicionales de calor. El principal problema para el clima mediterráneo es el periodo de amortización de los equipos, ya que durante una parte considerable del año no es necesario su uso.

\subsubsection{Climatización (calefacción y refrigeración)}

La aportación de calor y frío al espacio interior se efectúa por un sistema radiante dispuesto en el falso techo. Este sistema
${ }^{2}$ h-1 = ratio de renovación en volúmenes / hora. importante. 
permite trabajar con rangos de temperatura del agua más bajos (templados en invierno y menos fríos en verano) que permiten utilizar un porcentaje mayor de fuentes renovables e incrementan el rendimiento de los equipos de producción. Además, superficies radiantes permiten aumentar el rango de temperaturas de confort del aire del espacio, ya que la temperatura percibida del cuerpo humano es influenciada por partes iguales por la temperatura del aire y por las temperaturas de las superficies con las cuales intercambia radiación.

El proyecto LIMA no se centra en la eficiencia de los modelos de producción de calor i frío, que disponen ya de estudios exhaustivos, sino que su objetivo central es la reducción de la demanda. Por ello el modelo se ha desarrollado en base a sistemas de alto rendimiento, como los basados en redes urbanas de frío y calor, con plantas de cogeneración o trigeneración, biomasa o bombas de calor agua-agua con intercambio en masas de agua naturales (mar o ríos) o en sistemas geotérmicos de baja entalpia. El ahorro de emisiones de $\mathrm{CO}_{2 \text { eq }}$ para estos sistemas en comparación con sistemas convencionales descentralizados es significante - dependiendo de la tecnología utilizada y la fracción de incorporación de energías renovables, a parte de una serie de otras ventajas como la seguridad de servicio, de mantenimiento centralizado, etc.

\subsection{Simulación energética}

La simulación energética para la determinación de la demanda energética para calefacción, refrigeración y ventilación se ha efectuado con el programa THERMPLAN, de la consultora Doppelintegral $\mathrm{GmbH}$, expertos asociados al Centro de Investigación Aplicada en Tecnologías energéticas sostenibles (zafh.net) de la Universidad de Stuttgart, Alemania.

Para el cálculo de la demanda se ha considerado una ocupación de 3 personas, con una aportación de calor sensible de 50W por persona en estado de reposo y de $70 \mathrm{~W}$ en estado de actividad ligera (16). Como cargas internas debidas a cocina, horno eléctrico y electrodomésticos se ha utilizado principalmente los valores del programa de cálculo PHPP (Passivhaus Projektierungspaket) del Instituto Passivhaus, que detalla su distribución a los horarios de uso, diferenciado entre días laborales y fines de semana, así como datos de consumos obtenidos directamente de los fabricantes.

En la simulación energética se ha partido de un ratio de renovación de aire de ventilación forzada de $0,4 h^{-1}$ en régimen de calefacción y $0,52 \mathrm{~h}^{-1}$ en régimen de refrigeración, más un ratio adicional de $0,2 \mathrm{~h}^{-1}$ debido a infiltraciones.

Las condiciones de consigna, ocupación, carga interna y ventilación se indican en la siguiente tabla:

Tabla 2

Régimen de invierno, condiciones de consigna, ocupación, cargas internas y ventilación

\begin{tabular}{|c|c|c|c|c|c|c|}
\hline lunes a viernes & $\mathrm{h}$ & 00:00 - 06:00 & 06:00 - 09:00 & 09:00 - 18:00 & $18: 00-22: 00$ & $22: 00-24: 00$ \\
\hline consigna & ${ }^{\circ} \mathrm{C}$ & 17 & 20 & 15 & 20 & 17 \\
\hline ocupación & W & 150 & 210 & 0 & 210 & 150 \\
\hline cargas & W & 32 & 327 & 32 & 327 & 32 \\
\hline ventilación & $\mathrm{h}^{-1}$ & 0,6 & 0,6 & 0,2 & 0,6 & 0,6 \\
\hline sábado y domingo & $\mathrm{h}$ & 00:00 - 09:00 & 09:00 - 11:00 & $11: 00-14: 00$ & 14:00 - 19:00 & $19: 00-24: 00$ \\
\hline consigna & ${ }^{\circ} \mathrm{C}$ & 17 & 20 & 15 & 20 & 17 \\
\hline ocupación & $\mathrm{W}$ & 150 & 210 & 0 & 140 & 210 \\
\hline cargas & W & 32 & 327 & 32 & 527 & 32 \\
\hline ventilación & $h^{-1}$ & 0,6 & 0,6 & 0,2 & 0,6 & 0,6 \\
\hline
\end{tabular}

Tabla 3

Régimen de verano, condiciones de consigna, ocupación, cargas internas y ventilación

\begin{tabular}{|c|c|c|c|c|c|c|}
\hline lunes a viernes & $\mathrm{h}$ & 00:00 - 06:00 & 06:00 - 09:00 & 09:00 - 18:00 & 18:00 - 22:00 & $22: 00-24: 00$ \\
\hline consigna & ${ }^{\circ} \mathrm{C}$ & 26 & 26 & 32 & 26 & 26 \\
\hline ocupación & W & 150 & 210 & 0 & 210 & 150 \\
\hline cargas & $W$ & 32 & 327 & 32 & 327 & 32 \\
\hline ventilación & h-1 & 0,72 & 0,72 & 0,2 & 0,72 & 0,72 \\
\hline sábado y domingo & $\mathrm{h}$ & 00:00 -09:00 & 09:00 - 11:00 & $11: 00-14: 00$ & $14: 00-19: 00$ & 19:00 - 24:00 \\
\hline consigna & ${ }^{\circ} \mathrm{C}$ & 26 & 26 & 32 & 26 & 26 \\
\hline ocupación & W & 150 & 210 & 0 & 140 & 150 \\
\hline cargas & $W$ & 32 & 327 & 32 & 527 & 32 \\
\hline ventilación & h-1 & 0,72 & 0,72 & 0,2 & 0,72 & 0,72 \\
\hline
\end{tabular}


7. LIMA. Demanda horaria de calor y frío para 12 viviendas.
Los resultados de la demanda energética obtenidos, y que se detallan en la tabla siguiente, muestran un edificio de muy baja demanda. Es interesante destacar que la demanda de refrigeración es cuatro veces mayor que la de calefacción, y que estan última se produce casi exclusivamente debido a la ventilación y disminuye de modo significativo al incorporar un sistema de recuperación de aire.

Tabla 4

LIMA, demanda mensual de calefacción y refrigeración para 12 viviendas

\begin{tabular}{|lrrr|}
\hline & $\begin{array}{c}\text { Calefacción } \\
\text { sin recuperador }\end{array}$ & $\begin{array}{c}\text { Calefacción } \\
\text { con recuperador }\end{array}$ & $\begin{array}{c}\text { Refrigeración } \\
26^{\circ} \mathrm{C}\end{array}$ \\
\hline Enero & {$[\mathrm{kWh}]$} & {$[\mathrm{kWh}]$} & {$[\mathrm{kWh}]$} \\
\hline Febrero & $2.219,1$ & 777,6 & 0 \\
\hline Marzo & $1.614,6$ & 530,4 & 0 \\
\hline Abril & 56,2 & 1,4 & 0 \\
\hline Mayo & 6,6 & 0 & 0 \\
\hline Junio & 0 & 0 & $1.132,0$ \\
\hline Julio & 0 & 0 & $3.386,5$ \\
\hline Agosto & 0 & 0 & $1.778,9$ \\
\hline Septiembre & 0 & 0 & $1.469,2$ \\
\hline Octubre & 0 & 0 & 05,2 \\
\hline Noviembre & 0 & 0 & 0 \\
\hline Diciembre & 95,1 & 0 & $7.851,7$ \\
\hline & $1.644,9$ & & 9,16 \\
\hline $\mathrm{kWh} /$ año & $5,636,4$ & $1.937,5$ & 0 \\
\hline $\mathrm{kWh} / \mathrm{m}^{2} \cdot$ año & 6,57 & & 0 \\
\hline
\end{tabular}

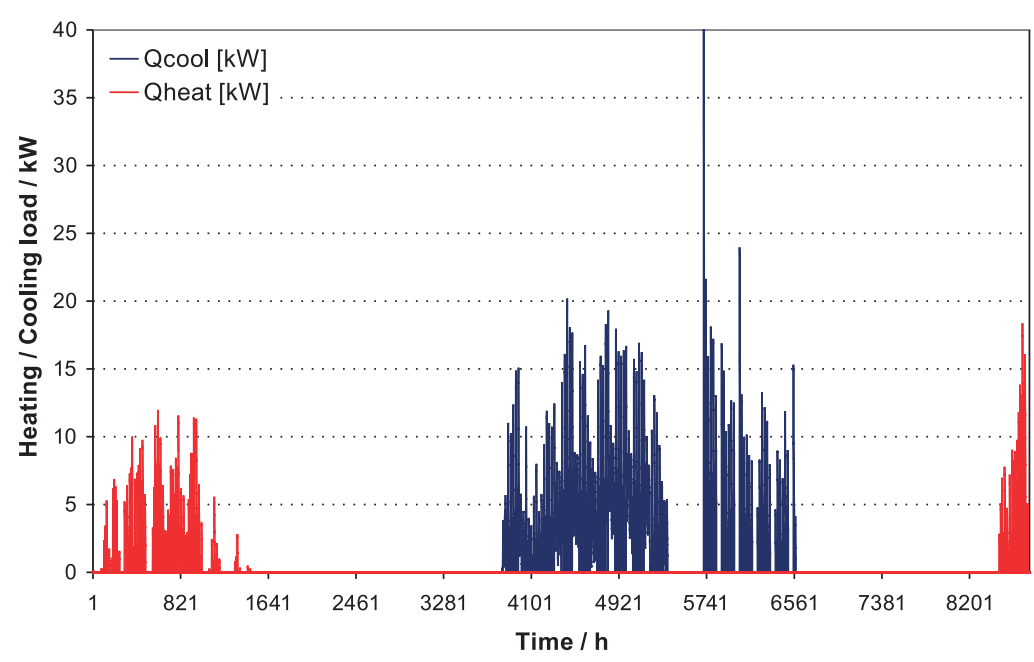

\section{PROCEDIMIENTO DE VERIFICACIÓN A TRAVÉS DE UN PROTOTIPO}

Para verificar los datos obtenidos en el cálculo se proyectó y construyó un prototipo experimental equivalente a la zona de día de una de las viviendas de LIMA 12. Para ello se contó con la participación de más de cuarenta empresas de diversos países que aportaron sus materiales y conocimientos. El prototipo se presentó en Construmat 2009, y posteriormente se trasladó a las instalaciones de la Escuela de Arquitectura e Ingeniería de La Salle, en Barcelona.

El prototipo se dotó de una central externa de producción de calor y frío en base de una bomba de calor aire-agua de alta eficiencia, para la producción de calor y frío, y un aporte adicional de calor a través de una instalación solar térmica. Esta instalación, que emula un sistema de calefacción y refrigeración central (edificio, manzana o distrito) dispone de dos circuitos, uno para climatización (calor y frío) y otro para producción instantánea de ACS, con intercambiadores agua-agua. Todas las aportaciones de energía se registran a través de contadores individuales de energía situados en la fachada del prototipo.

Durante 2010 se diseño e implementó el sistema de toma de datos, que combina sondas analógicas y digitales. Se analizan datos correspondientes a:

Climatologia: humedad relativa, temperatura y entalpía del aire exterior, radiación solar horizontal y en cada fachada, calidad del aire exterior $\left(\mathrm{CO}_{2}\right)$, velocidad y dirección del viento, y pluviometría.

Confort y salubridad: humedad relativa, temperatura y entalpía del aire interior en cuatro diferentes puntos, posición de las puertas (abierta/cerrada), posición de las protecciones solares, calidad del aire interior $\left(\mathrm{CO}_{2} \mathrm{y}\right.$ compuestos orgánicos volátiles-VOC).

Flujo de energía en los cerramientos: temperatura en cada capa de la fachada $\mathrm{SO}$ (4 sondas), humedad del compost de la cubierta, humedad en diferentes capas de la cubierta (2 sondas).

Recuperador de calor: humedad relativa, temperatura y entalpía del aire de retorno, expulsado, de captación y de impulsión hacia la sala (4 puntos), volumen del aire de expulsión y captación (2 puntos), posición recuperador/free-cooling, consumo eléctrico del ventilador del recuperador.

Sistema de calefacción/refrigeración: producción calor/frío, batería de calor on/off, techo radiante on/off, demanda de calor y frío (energía aportada por la batería de calor, energía aportada por el techo radiante), consumo eléctrico de la bomba del techo radiante.

Otros: energía consumida para la producción de ACS (bomba), iluminación, y electrodomésticos (4 contadores). 


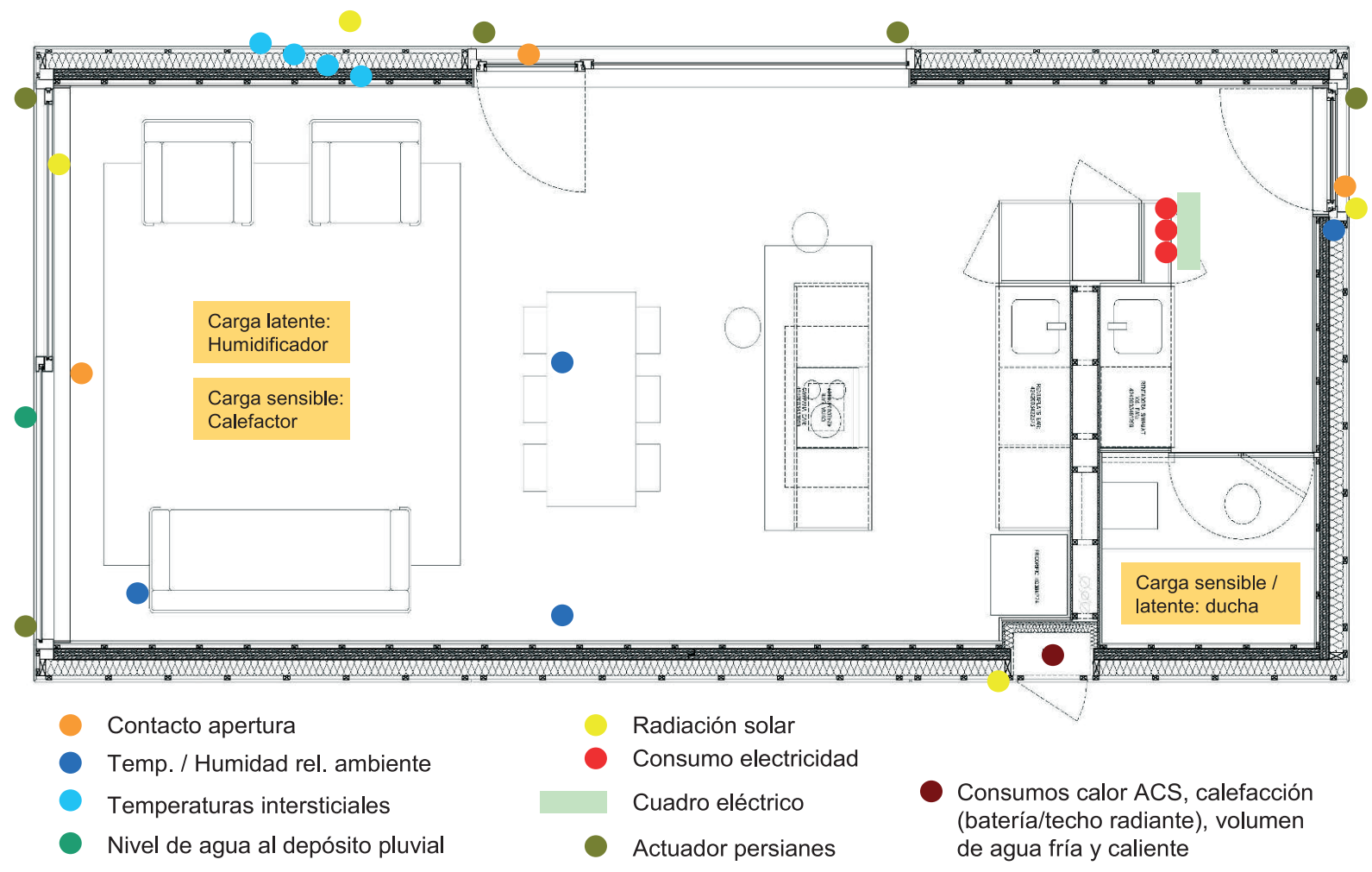

En total se trata de más de cien puntos de control que permiten monitorizar con gran detalle el funcionamiento del prototipo. Los datos aportados son almacenados en una base de datos SQL cada 15 minutos y los más significativos se visualizan en una página web, adaptando la resolución de los datos gráficamente representados (valores de $15 \mathrm{~min} /$ hora/día/mes) según el periodo de selección de muestra de datos.

El prototipo dispone además de un completo sistema de simulación de cargas internas y ocupación. Este sistema está formado por un calefactor que introduce, mediante un programa de control y una agenda, cargas térmicas que emulan el calor aportado por electrodomésticos y equipos, así como el calor sensible aportado por la ocupación. En paralelo un vaporizador de producción de vapor frío emula las aportaciones de calor latente debidas a la ocupación. Una vez completado el proceso se obtendrá un modelo real, el prototipo, y otro virtual, la simulación, con idénticas condiciones referentes a cargas internas y clima. Este doble modelo permitirá verificar el funcionamiento y la fiabilidad de los programas de simulación utilizados, mediante la simulación del prototipo en las condiciones meteorológicas reales del emplazamiento.

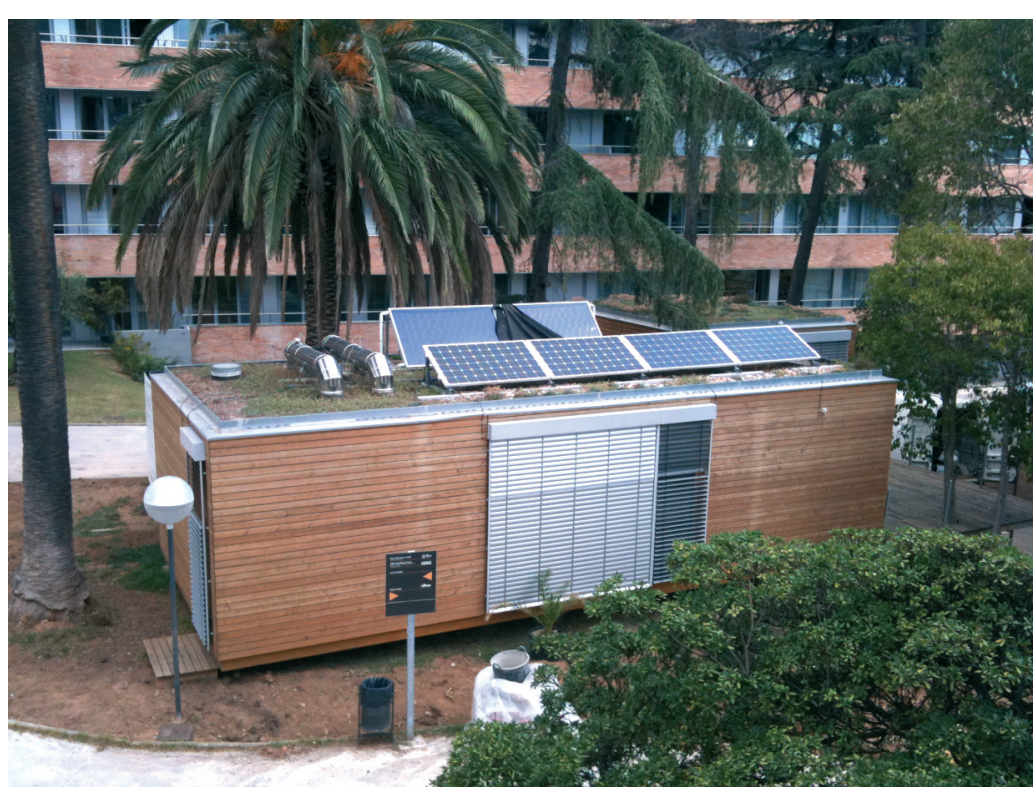

La verificación consta de dos fases.

En la primera fase (5-a) a realizar en los periodos de invierno i verano de 2011 se comprobará el funcionamiento del prototipo en condiciones estándar de uso, de acuerdo con el cuadro de ocupación y cargas internas definidos en la fase de simulación. Se analizaran: 


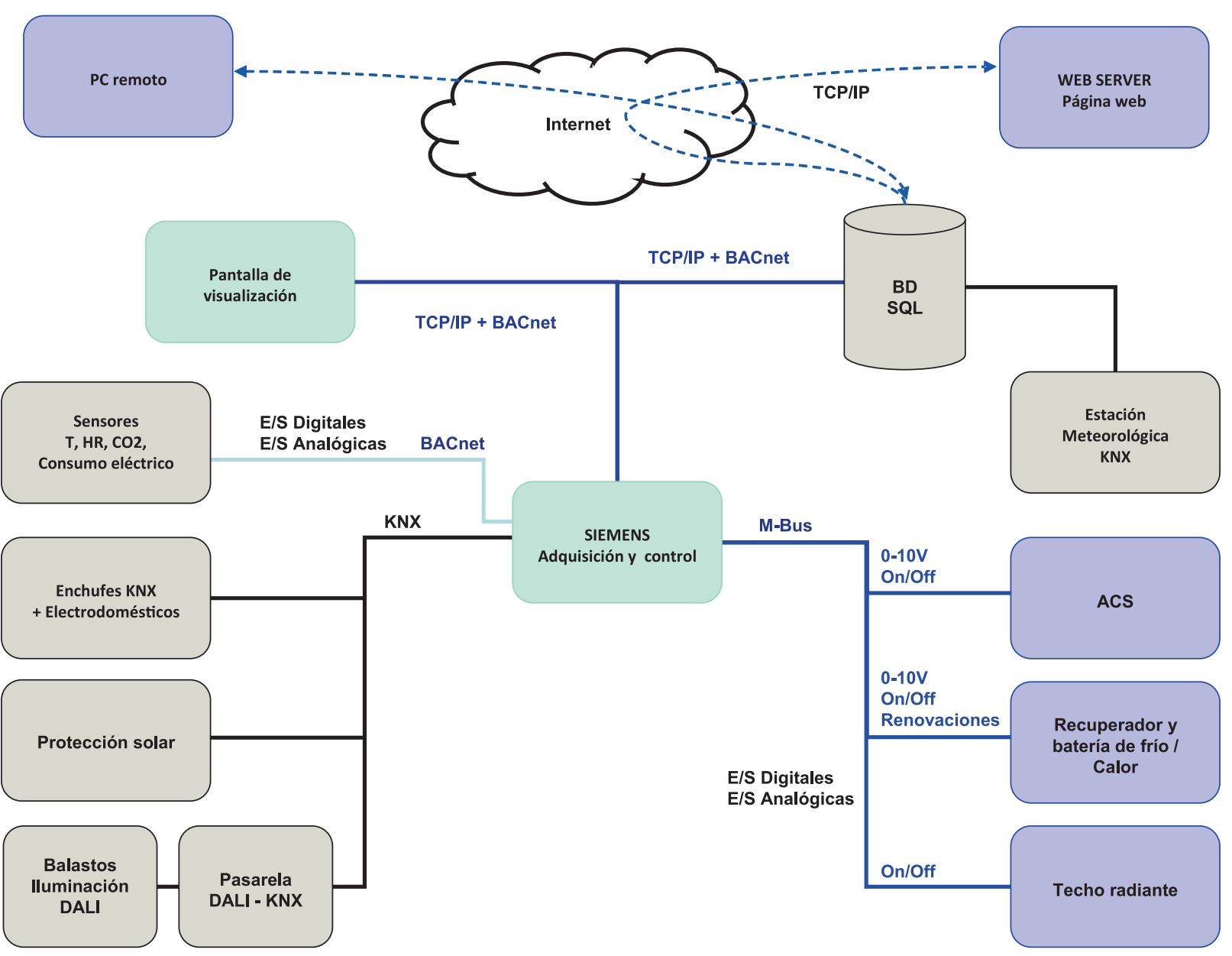

10. LIMA. Protocolo de control y monitorización y transmisión de datos.

\section{Energía}

- Demanda y consumo en uso $\left(\mathrm{kWh} / \mathrm{m}^{2} \cdot \mathrm{año}\right)$ y emisiones asociadas $\left(\mathrm{KgCO}_{2 \mathrm{eq}} / \mathrm{m}^{2} \cdot \mathrm{año}\right)$

- Funcionamiento de la envolvente térmica y los sistemas pasivos

- Rendimiento de los sistemas térmicos y eficiencia del recuperador entálpico

- Verificación de programas de simulación

En la segunda parte, a realizar entre 2012 y 2013, en condiciones de uso reales de una familia, se analizaran además de los datos anteriormente citados los siguientes: el ciclo completo del agua, y datos referentes a la biohabitabilidad como campos eléctricos y electromagnéticos, y COV debidos a los materiales:

\section{Energia}

- Demanda y consumo en uso $\left(\mathrm{kWh} / \mathrm{m}^{2} \cdot \mathrm{año}\right)$ y emisiones asociadas $\left(\mathrm{KgCO}_{2 \mathrm{eq}} / \mathrm{m}^{2} \cdot \mathrm{año}\right)$, con la incorporación de sistemas de aprovechamiento de inercia estacional para el condicionamiento del aire de ventilación (pozos provenzales)
- Funcionamiento de la envolvente térmica y los sistemas pasivos

- Rendimiento de los sistemas térmicos

- Verificación de programas de simulación

- Producción solar térmica y fotovoltaica

- Efectos del uso y las condiciones de consigna

Agua

- Captación de agua y periodo de atenuación

- Evapotranspiración de la cubierta verde

\section{Confort y biohabitabilidad}

- Control de calidad del aire ( $\mathrm{CO}_{2}$ y $\left.\mathrm{COV}\right)$

- Análisis de campos eléctricos y electromagnéticos

\section{CONCLUSIONES}

El proyecto LIMA establece los ámbitos de análisis para la definición de un edificio de muy bajo impacto ambiental en la climatología mediterránea y concibe un edificio de referencia para la verificación teórica de este 
modelo, así como un prototipo construido para la verificación práctica.

Los cálculos realizados sobre la energia incorporada y las emisiones de $\mathrm{CO}_{2 \text { eq }}$ asociados, en la producción y transporte de los materiales de construcción, incluidos los necesarios para el mantenimiento, dan un balance cero $\left(-0,63 \mathrm{kgCO}_{2} \cdot \mathrm{m}^{2} \cdot \mathrm{a}\right)$, aportando datos significativos sobre el impacto, especialmente debido a las emisiones de $\mathrm{CO}_{2 \text { eq, }}$ de esta fase del ciclo de vida de las edificaciones. En este sentido constituye un dato relevante la reducción de la energía incorporada al substituir el hormigón armado como elemento básico de la estructura por paneles de madera contralaminada.

Las simulaciones sobre demanda energética para climatización dan también resultados muy satisfactorios, con demandas de calefacción de 2,3 $\mathrm{kWh} / \mathrm{m}^{2}$.a, y de refrigeración de $9,2 \mathrm{kWh} / \mathrm{m}^{2} \cdot \mathrm{a}$. Ello permite afirmar que es viable técnicamente lograr un estándar de consumo energético muy próximo a cero.

En una primera fase de ensayo se comprobará principalmente el comportamiento térmico del prototipo construido en condiciones estandarizadas de uso.

Una segunda fase a realizar en los próximos años, permitirá verificar la aproximación de las simulaciones energéticas, así como la eficiencia y el retorno económico de diferentes sistemas pasivos y activos de reducción de la demanda de climatización (envolvente térmica, recuperador de calor, conductos provenzales, techo radiante, etc.).

\section{BIBLIOGRAFÍA}

(1) Intergovernmental Panel on Climate Change (IPCC). www.ipcc.ch.

(2) Stern, N.: Informe Stern, la verdad sobre el cambio climático, pp. 21-27, Ed. Paidós, Barcelona, 2007.

(3) European Building Performance Directive (EPBD) 2002/91/EC, adoptada por el Parlamento Europeo el 16/12/2002.

(4) Bates, Z. W.; Kundzewicz, B. C.; Wu, S. y Palutikof, J. P.: IPCC Technical papers 6: Cambios climáticos observados y proyectados en relación al agua, p. 27, IPCC Secretariat, Ginebra, 2008.

(5) Sabaté, J., Cuchí, A. et al.: Estudi sobre les possibilitats de reducció d'emissions de $\mathrm{CO}_{2}$ en habitatge social i la seva aplicació en una promoció de 60 habitatges a Can Vergonyós, Tossa de Mar. Departament de Territori i Sostenibilitat, Generalitat de Catalunya, 2006.

(6) Cuchí, A.; Wadel, G.; Rivas, P.: Cambio Global España 2020/50 - Sector Edificación. Ed.: Societat Orgánica amb Green Building Council España, Asociación Sostenibilidad y Arquitectura, p. 27, Centro Complutense de Estudios e Información Ambiental, Madrid, 2010.

(7) Winther, B. N. y Hestnes, A. G.: "Solar versus Green: the analysis of a Norwegian row house". Solar Energy, num. 66 (1999), pp. 387-393

(8) Sartori, I. \& Hestnes, A. G.: "Energy use in the life cycle of conventional and low-energy buildings". Energy and Buildings, num. 39 (2007), pp. 249-257.

(9) Feist, W.: Life cycle energy balances compared: low-energy house, passive house, selfsufficient house. International Symposium of CIB W67, Viena (1996), pp. 183-190.

(10) Sabaté, J.: "Arquitectura: un nou paradigma". 34 kg. de $\mathrm{CO}_{2}$, Departament de Territori I Sostenibilitat. Generalitat de Catalunya, 2009.

(11) Yates, M.; Linegar, M.; Dujic, B.: "Design of an 8 storey Residential Tower from KLH Cross Laminated Solid Timber Panels", Paper 299, Engineered Wood Products Association, 2008.

(12) Hegger, M.; Auch-Schwelk. V.; Fuchs, M.; Rosenkranz, T.: Construction Materials Manual, Ed. Birkhäuser, Basilea, 2007.

(13) Waltjen, T. et al.: "Bauteilkatalog Passivhaus - ökologisch bewertete Konstruktionen", Ed. IBO, Österreichisches Institut für Baubiologie und -ökologie, Wien, 2008.

(14) Ford, B.; Schiano-Phan, R.: El estándar Passivhaus en climas europeos templados. Resultados del proyecto europeo passive-on (EIE-04-091), enero 2005-septiembre 2007. www.passive-on.org.

(15) Peper, S.: Luftdichte Passivhäuser - Erfahrungen, Probleme, Lösungen und Dauerhaftigkeit; $6^{\text {a }}$ Conferència Passivhaus, Proceedings, Basilea, 2002.

(16) Leibundgut, H. J.: Die Eiserne Ration der Gebäudetechnik, Professur für Gebäudetechnik der ETH Zürich, 2009 